\title{
microRNAs modulate iPS cell generation
}

\author{
CHAO-SHUN YANG, ${ }^{1,2}$ ZHONGHAN LI, ${ }^{1,2}$ and TARIQ M. RANA ${ }^{1,2,3}$ \\ ${ }^{1}$ Program for RNA Biology, Sanford-Burnham Medical Research Institute, La Jolla, California 92037, USA \\ ${ }^{2}$ Department of Biochemistry and Molecular Pharmacology, University of Massachusetts Medical School, \\ Worcester, Massachusetts 01605, USA
}

\begin{abstract}
Although induced pluripotent stem cells (iPSCs) hold great promise for customized regenerative medicine, the molecular basis of reprogramming is largely unknown. Overcoming barriers that maintain cell identities is a critical step in the reprogramming of differentiated cells. Since microRNAs (miRNAs) modulate target genes tissue-specifically, we reasoned that distinct mouse embryonic fibroblast (MEF)-enriched miRNAs post-transcriptionally modulate proteins that function as reprogramming barriers. Inhibiting these miRNAs should influence cell signaling to lower those barriers. Here we show that depleting miR21 and miR-29a enhances reprogramming efficiency in MEFs. We also show that the p53 and ERK1/2 pathways are regulated by miR-21 and miR-29a and function in reprogramming. In addition, we provide the first evidence that c-Myc enhances reprogramming partly by repressing MEF-enriched miRNAs, such as miR-21 and miR-29a. Our results demonstrate the significance of miRNA function in regulating multiple signaling networks involved in iPSC generation. These studies should facilitate development of clinically applicable reprogramming strategies.
\end{abstract}

Keywords: miRNAs; miR-21, miR-29a; c-Myc-regulated miRs; iPS cells; p53; ERK1/2; miRNA-mediated reprogramming

\section{INTRODUCTION}

Embryonic stem (ES) cells can proliferate indefinitely and differentiate into various somatic cells. Therefore, ES cells are versatile tools for the study of early developmental processes and provide a promising source of tissues or cells useful for regenerative therapies. However, the derivation of human ES cells from embryos has been an ethical concern in the field. Recent breakthroughs in creating induced pluripotent stem (iPS) cells provide an alternative means to obtain ES-like cells without destroying embryos. iPS cells were first established by introducing four reprogramming factors (Oct3/4, Sox2, Klf4, and $c$-Myc) into mouse embryonic fibroblasts (MEFs) (Takahashi and Yamanaka 2006) or human fibroblasts (Takahashi et al. 2007). iPS cells have also been created following expression of Oct3/4, Sox2, Nanog, and Lin28 (Yu et al. 2007). Overall, iPS cells exhibit morphology, a transcriptome, and pluripotency similar to that of ES cells (Okita et al. 2007; Yu et al. 2007). However, retrovirus-mediated transgene expression and extremely low efficiency remain obstacles for their therapeutic application (Geoghegan and Byrnes 2008;

\footnotetext{
${ }^{3}$ Corresponding author.

E-mail trana@sanfordburnham.org.

Article published online ahead of print. Article and publication date are at http://www.rnajournal.org/cgi/doi/10.1261/rna.2664111.
}

Seifinejad et al. 2010; Yoshida and Yamanaka 2010). Moreover, although the combinatorial functions (Geoghegan and Byrnes 2008) and regulatory activity (Boyer et al. 2005; Chen et al. 2008; Kim et al. 2008; Sridharan et al. 2009) of the reprogramming factors have been established, the basic molecular mechanisms of each factor during the reprogramming remain unknown.

$\mathrm{c}-\mathrm{Myc}$, one of the four reprogramming factors (4F; Oct3/ 4, Sox2, Klf4, and c-Myc), plays crucial roles in cell proliferation and tumor development (Pelengaris et al. 2002). c-Myc is a key regulator of cytostasis and apoptosis through repression of the cyclin-dependent kinase (CDK) inhibitor p21 ${ }^{\mathrm{Cip} 1}$ (Seoane et al. 2002). By abrogating Miz-1 function and suppressing $\mathrm{p} 15^{\mathrm{INK} 4 \mathrm{~b}}, \mathrm{c}-\mathrm{Myc}$ plays a critical role in the immortalization of primary cells (Seoane et al. 2001). Many transcriptional functions of c-Myc require cooperation with Max or Miz-1 (Wanzel et al. 2003). As a proto-oncogene c-Myc greatly enhances reprogramming efficiency, although it is dispensable for reprogramming (Nakagawa et al. 2008; Sridharan et al. 2009). Therefore, defining molecular pathways downstream from c-Myc during reprogramming could enhance therapeutic application of iPS cells, without compromising reprogramming efficiency.

c-Myc reportedly acts to maintain ES cell renewal in part by regulating microRNA (miRNA) expression ( $\mathrm{Li}$ et al. 2009; Smith et al. 2010). miRNAs are 22-nucleotide (nt) 
noncoding small RNAs, which are loaded into RNA-induced silencing complex (RISC) to exert a global genesilencing function (Chu and Rana 2007). Expression of $m i R-141, m i R-200$, and $m i R-429$ is induced by c-Myc in ES cells to antagonize differentiation (Lin et al. 2009). c-Myc also promotes tumorigenesis by up-regulating the miR-1792 miRNA cluster (Dews et al. 2006) or by repressing known tumor suppressors, such as the let-7 family, miR15a/16-1, the miR-29 family, and miR-34a (Chang et al. 2008, 2009). Nonetheless, how c-Myc functions to initiate reprogramming is still unclear.

Overcoming barriers securing somatic cell identity and mediated by factors such as Ink4-Arf, p53, and p21 is a rate-limiting step in reprogramming (Banito et al. 2009; Hong et al. 2009; Judson et al. 2009; Kawamura et al. 2009; Marion et al. 2009; Utikal et al. 2009). Since miRNAs modulate target genes tissue-specifically (Farh et al. 2005; Rana 2007), we reasoned that distinct MEF miRNAs (Mayr and Bartel 2009) post-transcriptionally modulate proteins that function as reprogramming regulators. Inhibiting these miRNAs should influence cell signaling to lower those barriers.

Here, we show that depleting the abundant miRNAs miR-21 and miR-29a in MEFs enhances reprogramming efficiency by about 2.4-fold to threefold. We also report that c-Myc represses miRNAs miR-21 and miR-29a to enhance reprogramming of MEFs. Finally we report that miR-21 and miR-29a regulate 553 and ERK1/2 pathways by indirectly down-regulating p53 levels and ERK1/2 phosphorylation during the reprogramming process.

\section{RESULTS}

\section{Inhibition of miR-21 or miR-29a enhances reprogramming efficiency}

To determine whether inhibiting MEF-specific miRNAs lowers reprogramming barriers, we first analyzed MEF-enriched miRNAs and compared their levels with those seen in mouse ES cells. As shown in Figure 1A, let-7a, miR-21, and miR-29a were highly expressed in MEFs compared with ES cells. In contrast, miR-291 was highly abundant in ES but absent in MEFs (Fig. 1A). Next, we introduced miRNA inhibitors against let-7a, miR-21, and miR-29a into Oct4-EGFP MEFs (MEFs harboring Oct4-EGFP reporter) together with retroviruses expressing Oct3/4, Sox2, Klf4, and $c-M y c$ (OSKM). At day 14 post-transduction, cells treated with miR-21 inhibitors showed an about 2.4-fold increase in reprogramming efficiency compared with a nontargeting (NT) control (Fig. 1B). Similarly, reprogramming efficiency increased significantly by about threefold following inhibition of miR-29a (Fig. 1B). Under similar antagomir treatments as used for miR-29a or miR-21 inhibition, we observed a minor effect on OSKMreprogramming following let-7a inhibition (Fig. 1B). To further test whether miRNA inhibition enhances reprogram-
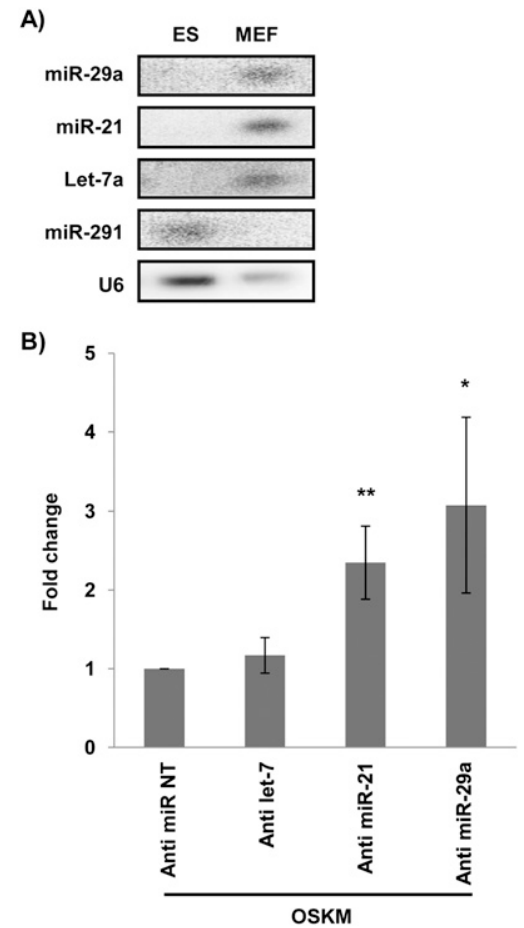

FIGURE 1. The inhibition of MEF-enriched microRNAs, miR-21 and miR-29a, enhances reprogramming efficiency. $(A)$ miR-29a, miR21 , and let7a are highly expressed in MEFs. Total RNAs were isolated from Oct4-EGFP MEFs and mouse ES cells and resolved by gel electrophoresis. Specific radioactive-labeled probes against the indicated miRNAs were used to detect expression. U6 snRNA served as a loading control. $(B)$ miRNA inhibition enhances reprogramming efficiency. Oct4-EGFP MEFs were transduced with OSKM as described in the Materials and Methods. GFP-positive colonies were identified and counted by fluorescence microscopy at day 14 after transduction. GFP+ colony number was normalized to the number of anti-miR nontargeting control treatment and is reported as foldchange. Error bars, SD of three independent experiments. ${ }^{\star} P$-value $<0.05 ;{ }^{*} P$-value $<0.005$.

ming with three factors in the absence of c-Myc, cells were transduced with the miRNA inhibitor together with Oct3/4, Sox2, and Klf4 (abbreviated as OSK), which reprograms cells at much lower efficiency than OSKM (Nakagawa et al. 2008). The number of OSK-reprogrammed iPS cell colonies increased in the presence of the miR-21 inhibitor relative to treatment with OSK alone (Supplemental Fig. S1). These results demonstrate that the depletion of the MEF-enriched miRNAs miR21 and miR-29 enhances 4F-reprogramming significantly and that blocking miR-21 moderately increases the efficiency of OSK reprogramming.

\section{c-Myc represses expression of miRNAs let-7a, miR-16, miR-21, miR-29a, and miR-143 during reprogramming}

Recent work indicates that the OSKM factors alter cell identity through both epigenetic and transcriptional mechanisms 
(Sridharan et al. 2009). Therefore, we hypothesized that the OSKM reprogramming factors could down-regulate MEFenriched miRNAs. To evaluate the potential effect of each reprogramming factor on miRNA expression, MEFs were transduced with various combinations of the OSKM factors and subjected to Northern blot analysis (Fig. 2A). Interestingly, Sox 2 alone induced expression level of miR-21, miR-29a, and let-7a by more than twofold, compared with the MEF control (Fig. 2B, left). Klf4 also induced miR-29a and let-7a by about 1.5 -fold and 1.8-fold, respectively (Fig. 2B, left). With Oct $3 / 4$ overexpression only, miRNAs did not change expression level (Fig. 2B, left). In contrast to Oct4, Sox2, and Klf4, the single factor c-Myc downregulated expression of miR-21 and miR-29a, the most abundant miRNAs in MEFs, by $\sim 70 \%$ of the MEF control (Fig. 2A,B, left).
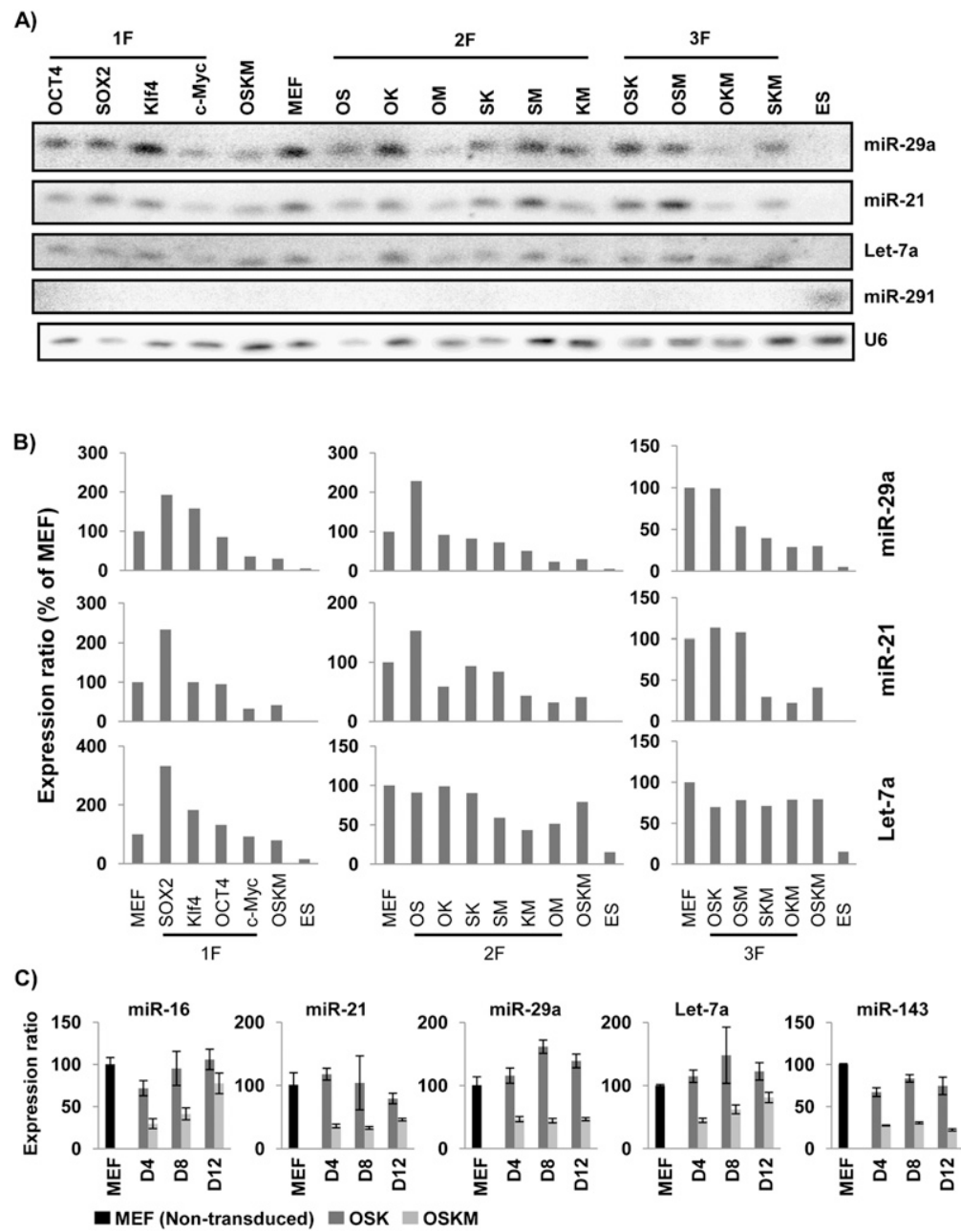

FIGURE 2. c-Myc is the primary repressor of MEF-enriched miRNAs during reprogramming. (A) Northern analysis of selected miRNAs at day 5 after reprogramming. Oct4-EGFP MEFs were transduced with a single factor or various combinations of reprogramming factors, as indicated. $1 \mathrm{~F}$ indicates one factor; $2 \mathrm{~F}$, two factors; $3 \mathrm{~F}$, three factors. OSKM indicates Oct $3 / 4$, Sox2, Klf4, and c-Myc. U6 is used as a loading control RNA. Total RNA from embryonic stem (ES) cells serve as negative control to MEF and transduced cells. Various probes were used to detect specific miRNAs as indicated on the right side. miR-291 blotting is a positive control for ES RNA. (B) Quantitative representation of miRNA expression in the presence of various reprogramming factors. Signal intensity was normalized to intensity of U6 snRNA. The expression ratio is calculated as the percentage of expression of each miRNA relative to expression in MEFs, which was arbitrarily set to $100 \%$. Various miRNAs were quantified (from panel $A$ ) and indicated on the right side. (C) Real-time RT-PCR analysis of selected miRNAs in Oct4-EGFP MEFs at various time points following OSK- or OSKM-reprogramming. RNA was isolated at the indicated day (D) after transduction for real time RT-PCR analysis. Signals were normalized to U6 and are shown as a percentage of miRNAs expressed in MEFs, which was arbitrarily set to 100 . Error bars, SD of two independent experiments.
Furthermore, among various combinations of two factors (2F) shown in Figure 2B (middle), inclusion of c-Myc enhanced decreases in all three miRNAs, including miR-21, miR-29a, and let-7a, by about $25 \%-80 \%$ (Fig. $2 \mathrm{~B}$, middle). Similar to the single-factor effect on miRNA expression, Sox 2 and Oct $3 / 4$ together increased miR-21 and miR-29a by 1.5-fold and 2.3-fold of the MEF control, respectively, while OK and SK had no obvious effects on miRNA expression (Fig. 2B, middle). Moreover, among various three-factor $(3 \mathrm{~F})$ combinations, the expression of miRNA-21 decreased by about $70 \%$ and $78 \%$ in SKM and OKM cells, respectively, relative to the expression seen in MEFs (Fig. 2B, right). Similarly, miR-29a expression decreased by about $48 \%-70 \%$ in $3 \mathrm{~F}$ combinations containing c-Myc (Fig. 2B, right). Inclusion of $\mathrm{c}-\mathrm{Myc}$ in $3 \mathrm{~F}$ combinations also slightly decreased let-7a levels (Fig. $2 \mathrm{~B}$, right). OSK without $\mathrm{c}-\mathrm{Myc}$ had little effect on miRNA expression (Fig. 2B, right). Therefore, these results strongly suggest that c-Myc plays an important role in regulating miRNA expression during the reprogramming.

To further confirm that $\mathrm{c}-\mathrm{Myc}$ is the primary factor antagonizing miRNA expression, cells were transduced with OSK with or without c-Myc, and miRNA expression was examined by real-time quantitative reverse transcription polymerase chain reaction (RT$\mathrm{qPCR}$ ) at various time points posttransduction. In contrast to OSK, OSKM transduction greatly decreased expression of let-7a, miR-16, miR-21, miR-29a, and miR-143 during reprogramming (Fig. $2 \mathrm{C}$ ), indicating that c-Myc plays a predominant role in regulating expression of MEF-enriched miRNAs, including the most abundant ones, let-7a, miR-21, and miR-29a. These data also suggest that 
c-Myc boosts reprogramming, in part, through miRNA down-regulation.

\section{c-Myc regulates miRNA expression at transcriptional level during reprogramming}

c-Myc has been shown to influence miRNA expression in multiple human and mouse cancer models (Chang et al. 2008, 2009), and Lin28b is one of the key intermediate modulators to post-transcriptionally regulate let-7 biogenesis (Chang et al. 2009). Therefore, we examined whether Lin28b-mediated regulation of miRNA expression plays a role during reprogramming. To address this question, we collected reprogrammed cells at various time intervals from days 3-15 after transduction of reprogramming factors. RT-qPCR analysis showed that mRNA expression of Lin28a and Lin28b were undetectable in MEF and during the reprogramming process, while ES cells exhibited a high level of Lin28a and Lin28b expression (Supplemental Fig. $\mathrm{S} 3 \mathrm{~A}, \mathrm{~B})$. Control marker gene expression analysis established the successful progression of reprogramming process where the levels of Thyl and Fibrillin-2 were downregulated and Nanog and Fbox 15 were up-regulated (Supplemental Fig. S3C). These findings strongly suggest that c-Myc regulation of miRNAs is Lin28-independent.

Next, to determine whether the c-Myc effect on MEFenriched miRNAs is post-transcriptional, we examined miRNA biogenesis by Northern blotting and quantified the amounts of pre-miRNAs and mature miRNAs. Our analysis showed that the ratio between pre-miRNA and mature miRNA in the presence of OSKM (Supplemental Fig. S4) was not changed, suggesting that the miRNA maturation process of miR-21 and miR-29a was not compromised by c-Myc during reprogramming. Altogether, these data show that the c-Myc-mediated downregulation of miR-21 and miR-29a is Lin28a/b-independent and occurs at the transcriptional level.

\section{iPS cells derived via miRNA depletion attain pluripotency}

To investigate whether blocking miR-21 or miR-29a compromises iPS cell pluripotency, we derived iPS cells treated with miR antagomirs and evaluated them for pluripotency (Li et al. 2011). Since OSKM-derived iPS cells were already well characterized in numerous studies, we decided to thoroughly examine our OSKM/anti miR-29a and OSKM/anti miR21 iPS cells, as well as OSK/anti miR21 clones. First, iPS cells were manually picked $\sim 2$ wk after reprogramming and were expanded to examine morphology and expression of ES-specific markers. Cells exhibited an ES-like morphology and a highly expressed Oct4-EGFP (Fig. 3A), indicating establishment of endogenous ES cell signaling. In addition, anti-miR-derived iPS cells expressed ES cell-specific markers, including Nanog and SSEA1, and exhibited alkaline phosphatase activity (Fig. 3A). To test whether those iPS cells showed pluripotent potential comparable to that of ES cells, those iPS cells were induced to form embryoid bodies (EBs) (Fig. 3B; Supplemental Movie S1) or were injected into nude mice (Fig. 3C) and allowed to differentiate into various tissues. After $2 \mathrm{wk}$ of in vitro differentiation, typical cell types derived from all three germ layers were observed (Fig. 3B; Supplemental Movie S1). Teratoma tumors, formed 3 wk after injection, were subjected to histopathologic analysis. Various tissues originating from all three germ layers (Fig. 3C) were generated, confirming that anti-miR-derived iPS cells obtained pluripotency. To use the most stringent test of pluripotency, iPS cells were injected into embryonic day (E) 3.5 blastocysts to create chimeric mice. Mouse derived from anti miR-29a iPS cells showed a significant $\sim 15 \%$ black coat color attributable to iPS cells (Fig. 3D). Since OSK in combination with miR-21 inhibitors resulted in high reprogramming efficiency (Supplemental Fig. S1), we also determined the pluripotency of OSK/anti miR-21 iPS cells by chimera analysis. Mouse generated from OSK/anti miR-21 iPS cells showed $\sim 25 \%$ black coat color (Fig. 3D). These data show that depleting miR-21 and miR-29a had no adverse effect on pluripotency of derived iPS cells.

\section{Inhibiting miR-29a down-regulates p53 through p85 $\alpha$ and CDC42 pathways}

To understand mechanisms underlying miR-29a's effect on reprogramming, we first examined expression of p85 $\alpha$ and CDC42, which are reportedly direct targets of miR-29 in HeLa cells (Park et al. 2009). To do so, we transfected miRNA inhibitors into MEFs and analyzed $\mathrm{p} 85 \alpha$ and CDC42 protein expression by Western blot at day 5 after transfection. As expected, $\mathrm{p} 85 \alpha$ and $\mathrm{CDC} 42$ protein levels increased slightly following the miR-29a block, whereas a let-7a inhibitor had little effect (Fig. 4A,B). The transformation related protein 53 (Trp53 or p53) is also reportedly a direct target of $\mathrm{p} 85 \alpha$ and CDC42 (Park et al. 2009). Therefore, we asked whether p53 is indirectly regulated by miR-29a in MEFs as well. To test that, MEFs were transfected with miRNA inhibitors and harvested 5 $\mathrm{d}$ for immunoblotting to evaluate expression of p53. p53 protein levels decreased by $\sim 30 \%$ (Fig. $4 \mathrm{~A}, \mathrm{~B}$ ) following miR-29a inhibition but were not altered by the NT control or by let-7a inhibition. Significantly, depleting miR-21 also released $\mathrm{p} 85 \alpha$ and CDC42 protein repression, and consequently, the levels of p85 $\alpha$ and CDC42 increased, which resulted in down-regulation of p53 expression by $\sim 25 \%$ (Fig. 4A,B).

To further confirm that p53 levels decrease with inhibition of miR-21 or miR-29a during reprogramming, we examined p53 expression at reprogramming day 5 by Western blot analysis. To initiate reprogramming we introduced miRNA inhibitors together with OSKM. Consistent 
A)

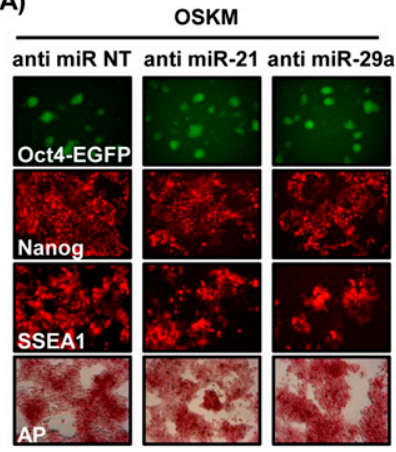

C)
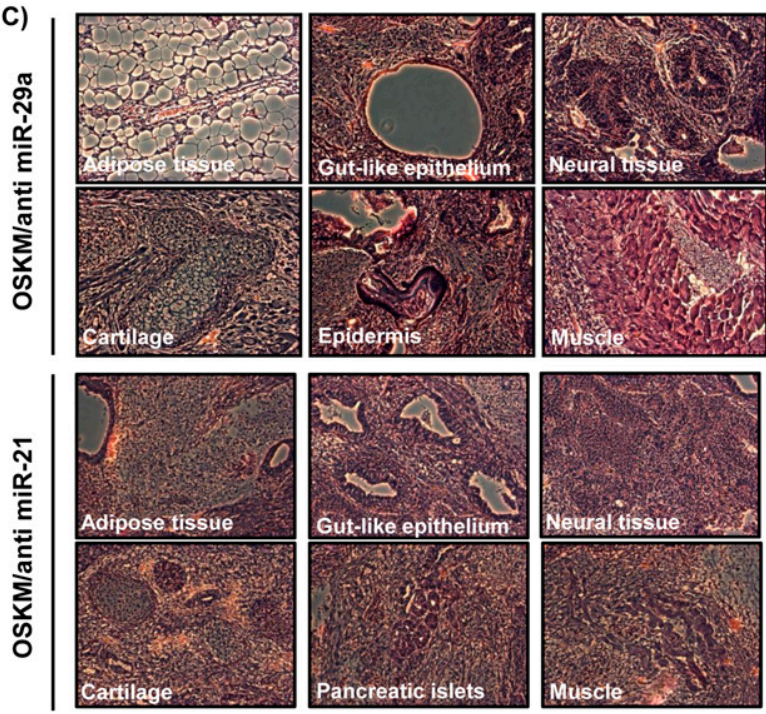

FIGURE 3. Mouse iPS cells derived with miR-21 and miR-29a inhibitors are pluripotent. $(A)$ Staining with ES cell markers of OSKM/anti miR-29a or miR-21 iPS cells. GFP+ colonies derived following OSKM and various miR inhibitor treatments were picked for further analysis. Representative colonies expressing the embryonic stem cell markers Nanog and SSEA1 are shown. Endogenous Oct $3 / 4$ was also activated, as indicated by the EGFP expression. Strong alkaline phosphatase (AP) activity is shown as one of the ES markers. Anti-miR NT (nontargeting) serves as miR inhibitor control. (B) In vitro differentiation of OSKM/anti miR29a or miR-21 iPS cells. Embryoid bodies were formed in vitro and cultured for 2 wk. Cells were fixed and stained with anti- $\alpha$ fetoprotein (for mesoderm) and anti- $\beta$-tubulin III (for ectoderm). Nuclei are shown as counter stain by Hoescht staining. $(C)$ Teratoma formation analysis of OSKM/anti miR-29a or miR-21 iPS cells. We injected $1.5 \times 10^{6}$ iPSCs subcutaneously into athymic nude female mice. Tumor masses were collected at $3 \mathrm{wk}$ after injection and fixed for histopathologic analysis. Various tissues derived from three germ layers were identified, including gut-like epithelium and pancreatic islet-like structure (endoderm); adipose tissue, cartilage, and muscle (mesoderm); and neural tissue and epidermis (ectoderm). (D) Chimera analysis of OSKM/anti miR-29a and OSK/anti miR-21 iPS cells. Eight to 14 iPS cells were injected into E3.5 mouse blastocysts. iPS cell contribution to each chimera was estimated by assessing black coat color and is shown as a percentage.

with observations in MEFs alone, p53 protein levels decreased by $\sim 25 \%$ or $\sim 40 \%$ following miR-21 or miR-29a depletion, respectively, during reprogramming, compared with OSKM controls (Fig. 4C). In summary, our data showed that blocking miR-29a reduced $\mathrm{p} 53$ protein levels by about $30 \%-40 \%$ through $\mathrm{p} 85 \alpha$ and CDC42 pathways during reprogramming. In addition, depletion of miR-21 had a similar effect on both $\mathrm{p} 85 \alpha$ and CDC42 and lowered p53 protein levels by about $25 \%$ to about $30 \%$.
Inhibition of miR-29a enhances reprogramming efficiency through p53 down-regulation

It was recently reported that p53 deficiency can greatly increase reprogramming efficiency (Banito et al. 2009; Hong et al. 2009; Judson et al. 2009; Kawamura et al. 2009; Marion et al. 2009; Utikal et al. 2009). Since depleting miR-29a significantly decreased p53 levels and increased reprogramming efficiency by about threefold (Fig. 1), we asked whether the effect of miR-29a knockdown is mediated primarily by p53 down-regulation. To that end, we transfected p53 siRNA and/or the miR29a inhibitor into Oct4-EGFP MEFs together with OSKM to initiate reprogramming. Down-regulation $(\sim 80 \%)$ of p53 by small interfering RNA (siRNA) had a similar positive effect on reprogramming efficiency as did miR-29a inhibition (Fig. 4D). We did not observe an increase in reprogramming efficiency when $\mathrm{miR}$ inhibitors were added in the presence of p53 siRNA (Fig. 4D). These results suggest that inhibition of miR29a acts, in part (see below), through down-regulation of p53 to increase reprogramming efficiency.

\section{Inhibition of miR-21 and miR-29a decreases phosphorylation of ERK1/2, but not GSK3 3 , to enhance reprogramming}

miR21 reportedly activates MAPK/ERK through inhibition of the sprouty homolog 1 (Spry1) in cardiac fibroblasts (Thum et al. 2008). Blocking MAPK/ ERK activity promotes reprogramming of neural stem cells (Silva et al. 2008) and secures the ground state of ESC self-renewal (Nichols et al. 2009; Ying et al. 2008). Therefore, we asked whether miR-21 regulates the MAPK/ERK pathway during reprogramming by evaluating ERK1/2 phosphorylation in MEFs following the introduction of miRNA inhibitors. To test that, MEFs were transfected with miRNA inhibitors and then harvested for Western blot analysis to determine the phosphorylated ERK1/2 level. Western blot analysis showed that blocking miR-21 significantly decreased by $\sim 45 \%$ the ERK1/2 phosphorylation relative to the NT controls, while let-7a inhibitors had no effect (Fig. 5A). 
A)

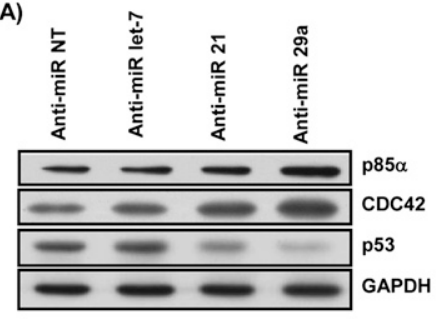

C)

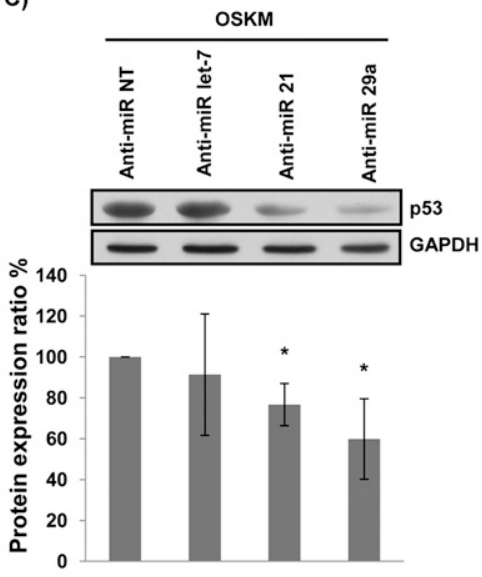

B)

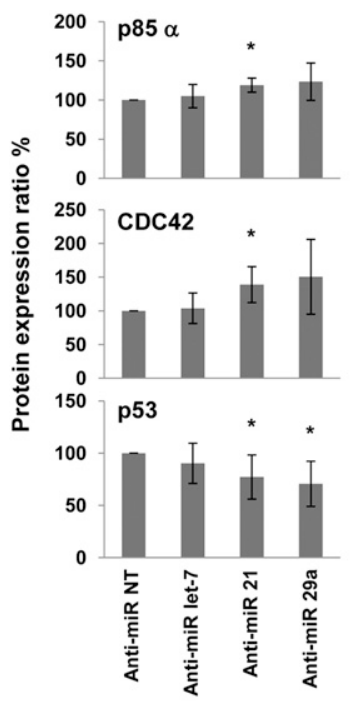

D)

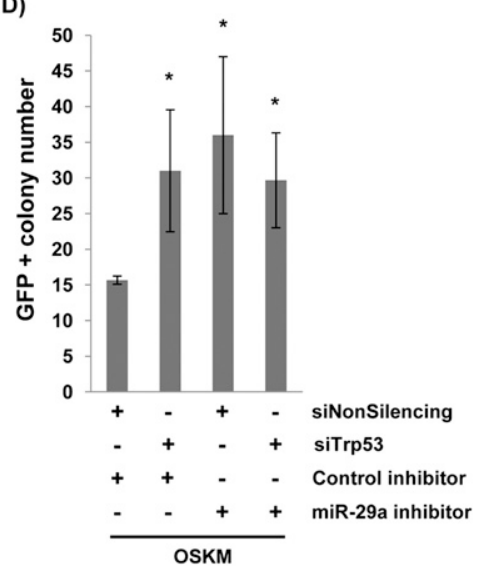

FIGURE 4. Inhibition of miR-21 or miR-29a enhances iPS cell reprogramming by decreasing p53 protein levels and up-regulating p $85 \alpha$ and CDC42 pathways. (A) Western analysis of expression of $\mathrm{p} 53, \mathrm{CDC} 42$, and $\mathrm{p} 85 \alpha$ following inhibition of various miRNAs. We transfected $1 \times 10^{5}$ Oct4-EGFP MEFs with the indicated miRNA inhibitors. Cells were harvested and analyzed $5 \mathrm{~d}$ later. $(B)$ Quantitative representation of protein expression in the presence of indicated miR inhibitors. Signal intensity was normalized to GAPDH intensity and is shown as a percentage relative to expression in control (NT) cells, which was set arbitrarily to 100. Error bars, SD of at least three independent experiments. ${ }^{\star} P$-value $<0.05$. (C) Immunoblot analysis of p53, CDC42, and p85 $\alpha$ expression following inhibition of various miRNAs and OSKM transduction. We transfected $1 \times 10^{5}$ Oct4-EGFP MEFs with the indicated miRNA inhibitors. Cells were harvested $5 \mathrm{~d}$ later and analyzed by immunoblot. Signal intensity was normalized as described in $B$. Error bars, SD of at least three independent experiments. ${ }^{\star} P$-value $<0.05$. (D) Depleting miR-29a or p53 enhances reprogramming efficiency. We transfected $4 \times 10^{4}$ Oct4EGFP MEFs with the indicated siRNAs and miRNA inhibitors, as well as OSKM reprogramming factors. GFP-positive cells were counted at day 12 after transduction. Error bars, SD of at least three independent experiments. ${ }^{*} P$-value $<0.05$.

demonstrate that depleting miR-21 and miR-29a down-regulates phosphorylation of ERK1/2 by modulating Spry1 protein levels.

To address whether ERK1/2 downregulation enhances reprogramming efficiency, we introduced siRNAs targeting ERK1 or ERK2 into Oct4-EGFP MEFs in the course of 4F-reprogramming. Depletion of either ERK1 or ERK2 significantly enhanced the generation of mature iPS cells (Fig. 5C). As expected, our data showed that miR-21 acts as an inducer of ERK1/2 activation in MEFs, since blocking miR-21 reduced ERK1/2 phosphorylation. Depleting miR29a also significantly diminished ERK1/2 phosphorylation. These results strongly suggest that miR-21 and miR-29a regulate ERK1/2 activity to modulate reprogramming efficiency (Fig. 5A-C).

The GSK3 $\beta$ pathway also represses ES self-renewal and reprogramming of neural stem cells (Ying et al. 2008). Depleting GSK3 $\beta$ with siRNA greatly increased mature iPS cell generation (Fig. 5C). Therefore, we asked whether miRNA depletion regulated GSK3 $\beta$ activation. Immunoblotting showed that blocking miRNAs in Oct4-EGFP MEFs had no significant effect on GSK3 $\beta$ activation (Fig. 5D). We then asked whether miRNA depletion alters apoptosis or cell proliferation during reprogramming by using flow cytometry to assess cell viability and replication rate. Blocking miRNA-21, miRNA-29a, or let-7 during reprogramming with OSKM did not alter apoptosis or proliferation rates (Supplemental Fig. S2). Overall, our results demonstrate that miR-29a and miR-21 modulate p53 and ERK1/2 pathways to regulate iPS cell reprogramming efficiency.
Interestingly, depleting MEFs of miR-29a also significantly reduced ERK1/2 phosphorylation by $60 \%$ relative to the NT control (Fig. 5A). Next we determined whether miR-21 and miR-29a affected ERK1/2 phosphorylation by altering Spry1 levels. We depleted miR-21 or miR-29a in MEFs by transfecting various miRNA inhibitors, and quantified Spry1 expression levels by immunoblotting. Our results showed that inhibiting miR-21 and miR-29a enhanced Spry1 expression levels (Fig. 5B). Therefore, our data

\section{DISCUSSION}

To develop alternatives for transgenes currently used for induced reprogramming, it is crucial to understand how signaling pathways are regulated by these factors. This is the first report to show that c-Myc represses MEF-enriched miRNAs, such as miR-21, let-7a, and miR-29a, during reprogramming (Fig. 1). Depleting miR-29a with inhibitors decreased p53 protein levels most likely by releasing p $85 \alpha$ 


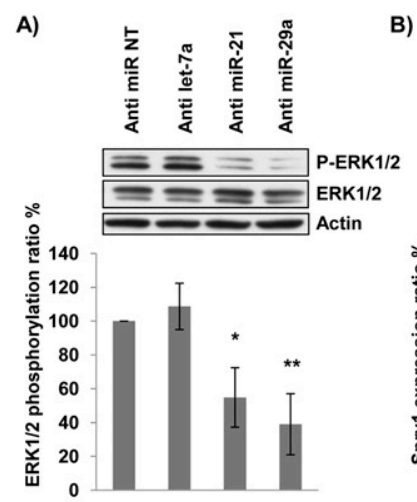

D)

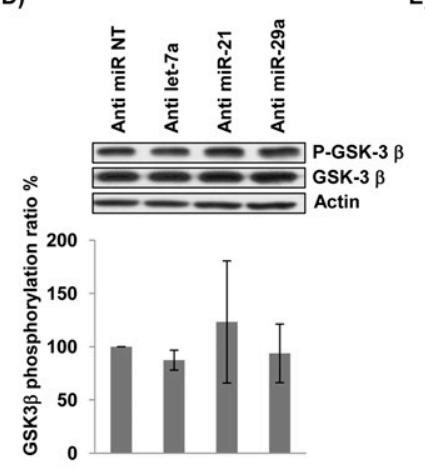

B)

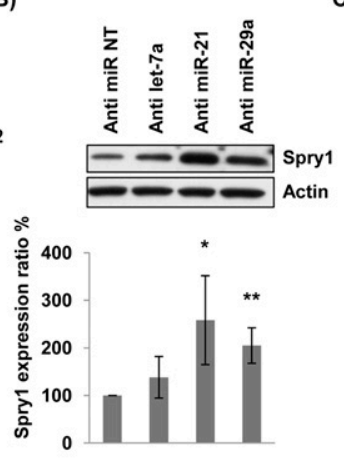

E)

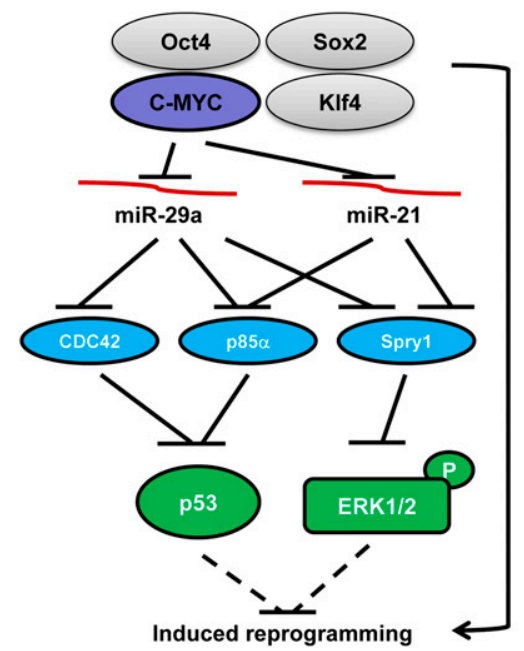

FIGURE 5. Depleting miR-21 and miR-29a promotes reprogramming efficiency by downregulating the ERK1/2 pathway. (A) Western analysis of phosphorylated and total ERK1/2 following inhibition of various miRNAs in MEFs. We transfected $1 \times 10^{5}$ Oct4-EGFP MEFs with the indicated miRNA inhibitors, harvested $5 \mathrm{~d}$ later, and immunoblotted. Signal intensity normalized to actin and shown as percentage relative to expression of anti-miR NT control. Error bars, SD of three independent experiments. ${ }^{*} P$-value $<0.05 ;{ }^{*} P$-value $<0.005$. (B) Western blot analysis of Spryl expression ratio shows that depleting miR-21 and miR-29a increases Spryl protein levels. MEFs were transfected with various miRNA inhibitors as indicated. Cells were harvested at day 5 after transfection for Western blot analysis. Signal intensity normalized to actin and shown as described in $A$. Error bars, SD of three independent experiments. ${ }^{\star} P$-value $<0.05 ;{ }^{\star} P$-value $<0.005$. (C) Fold-change in reprogramming efficiency following ERK1/2 or GSK3 $\beta$ knock-down. We transfected $4 \times 10^{4}$ Oct4-EGFP MEFs with the indicated siRNAs, as well as OSKM. GFP-positive cells were counted 2 wk later. Transfection with siNT serves as control for the reprogramming efficiency. Error bars, SD of three independent experiments. ${ }^{\star} P$-value $<0.05 ;{ }^{*} P$-value $<0.005$. $(D)$ Western analysis of phosphorylated and total GSK-3 $\beta$ following inhibition of various miRNAs in MEFs. We transfected $1 \times 10^{5}$ Oct4-EGFP MEFs with the indicated miRNA inhibitors, harvested 5 $\mathrm{d}$ later, and analyzed by immunoblot. Signal intensity normalized as described in $A$. Error bars, $\mathrm{SD}$ of three independent experiments. (E) Schematic representation showing that c-Myc enhances reprogramming by down-regulating the MEF-enriched miRNAs, miR-21 and miR29a. The p53 and ERK1/2 pathways function as barriers to reprogramming, and miR-21 and miR-29a indirectly activate those pathways through down-regulating CDC42, p $85 \alpha$, and Spryl. The cross-talk between miR-21/p53 and miR-29a/ERK1/2 pathways is also shown. c-Myc represses expression of these miRNAs and in turn compromises induction of ERK $1 / 2$ and p53. The dotted lines indicate p53 and ERK1/2 effects on iPS generation.

and CDC42 repression (Fig. 4). In addition, depleting miR21 decreased ERK1/2 phosphorylation (Fig. 5). Interestingly, we found that miR-21 inhibition reduced p53 protein levels and that inhibiting miR-29a also reduced ERK1/2 phosphorylation level. Both p53 and ERK1/2 signaling

antagonizes reprogramming (Banito et al. 2009; Hong et al. 2009; Judson et al. 2009; Kawamura et al. 2009; Marion et al. 2009; Silva et al. 2008; Utikal et al. 2009). Blocking miR-21 and miR-29a or knockdown of p53 and ERK1/2 can enhance reprogramming efficiency (Figs. 4, 5). Thus, we propose that c-Myc facilitates reprogramming in part by suppressing the MEF-enriched miRNAs, miR-21 and miR-29a, that act as reprogramming barriers through induction of p53 protein levels and ERK1/2 activation (Fig. 5E).

Forced expression of ES-specific miRNAs of the miR-290 family can replace c-Myc to promote reprogramming (Judson et al. 2009). c-Myc also binds the promoter region of the miR290 cluster (Chen et al. 2008; Judson et al. 2009). However, early expression of the c-Myc transgene is effective to initiate reprogramming but dispensable at the transition stage or later in mature iPS cells (Sridharan et al. 2009), where miR-290 clusters start to express. Therefore, it is unlikely that c-Myc promotes early stages of reprogramming through activating the miR-290 family.

We also found that expression level of MEF-enriched miRNAs, including miR-29a, miR-21, miR-143, and let-7a, decreases when c-Myc is introduced for reprogramming. c-Myc has a profound transcriptional effect (Wanzel et al. 2003) on miRNAs in promoting tumorigenesis (Chang et al. 2008, 2009) or sustaining the pluripotency ground state (Lin et al. 2009; Smith et al. 2010). Therefore, c-Myc repression of miRNA expression is the likely mechanism underlying reprogramming.

miR-21 acts as positive mediator to enhance fibrogenic activity through the TGF $\beta 1$ (Liu et al. 2010) and ERK1/2 (Thum et al. 2008) pathways, both of which have been shown to influence reprogramming and the ES cell ground state (Ichida et al. 2009; Nichols et al. 2009; Ying et al. 2008). Notably, among validated miR-29a targets, the protein level of p53 is indirectly induced by miR-29a (Park et al. 2009). In addition, recent studies show that the Ink4-Arf/ p53/p21 pathway compromises reprogramming and that p53 deficiency greatly enhances reprogramming efficiency (Banito et al. 2009; Hong et al. 2009; Judson et al. 2009; 
Kawamura et al. 2009; Marion et al. 2009; Utikal et al. 2009). Thus these signaling pathways are likely the primary barriers to the reprogramming process.

Depleting the c-Myc-targeted miRNAs, miR-21 and miR-29a, enhanced reprogramming efficiency about 2.4fold to about threefold (Fig. 1), suggesting that MEFenriched miRNAs also function as reprogramming barriers. Let-7 inhibition has been recently reported to enhance reprogramming (Melton et al. 2010); however, in several attempts we observed a minor effect in reprogramming when let-7 was inhibited by antagomirs (Fig. 1). Moreover, our data showed that the induction of p53 during reprogramming was compromised by miR-29a inhibition, enhancing reprogramming efficiency. Similarly, reprogramming can be greatly promoted by depleting either miR-21 or ERK1/2. c-Myc is a major contributor to the early stage of reprogramming and is not required to sustain the process at transition and late stages (Sridharan et al. 2009), indicating that c-Myc-regulated miRNAs may be employed to initiate high efficiency reprogramming.

c-Myc reportedly directly binds to and represses the miR-29a promoter (Chang et al. 2008). However, further studies are needed to understand how c-Myc regulates miR-21 expression. Our data show that c-Myc can be only partially replaced by depleting miR-21 and suggest that c-Myc has other functions in reprogramming. Thus the regulation of multiple pathways or wide repression of MEF-enriched miRNAs may be required to replace c-Myc function during reprogramming.

In summary, here we show that c-Myc reduces the threshold for reprogramming by decreasing p53 levels and antagonizing ERK1/2 activation through miR-21 and miR-29a down-regulation. Additionally, factors downstream from c-Myc may serve as targets for manipulation by siRNA, miRNA, or small molecules, to improve reprogramming. These approaches could be extended to replace all four reprogramming factors.

\section{MATERIALS AND METHODS}

\section{MEF derivation}

Oct4-EGFP MEFs were derived from the mouse strain B6;129S4-

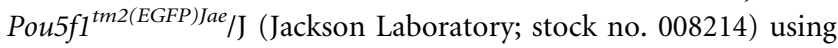
the protocol provided on the WiCell Research Institute website (http://www.wicell.org/). Oct4-EGFP MEFs were maintained in MEF complete medium (DMEM with 10\% FBS, nonessential amino acids, L-glutamine, but without sodium pyruvate).

\section{Reprogramming using retrovirus}

Reprogramming was conducted as described (Takahashi and Yamanaka 2006). In brief, $4 \times 10^{4}$ Oct4-EGFP MEFs were transduced with pMX retroviruses to overexpress Oct4, Sox2, Klf4, and c-Myc (Addgene). Two days later, transduced Oct4EGFP MEFs were fed with ES medium (DMEM with 15\% ES- screened FBS, nonessential amino acids, L-glutamine, monothioglycerol, and $1000 \mathrm{U} / \mathrm{mL} \mathrm{LIF})$, and the media were changed every other day. Reprogrammed pluripotent stem cells (defined as EGFP+ iPSC colonies) were scored by fluorescence microscopy $\sim 2$ wk after transduction, unless otherwise stated. To derive iPSCs, EGFP+ colonies were manually picked under a stereo microscope (Leica).

\section{miRNA inhibitor or siRNA transfection}

Inhibitors of let-7a, miR-21, and miR-29a miRNAs were purchased from Dharmacon. We transfected $4 \times 10^{4}$ Oct4-EGFP MEFs with lipofectamine and inhibitors according to manufacturer's instruction (Invitrogen). Three to $5 \mathrm{hr}$ later, the medium was discarded and replaced with MEF complete medium; for reprogramming, retrovirus encoding reprogramming factors (Oct4, Sox2, Klf4, and c-Myc) was added and the medium was changed to complete medium the next day. Inhibitors or siRNAs were introduced again at day 5 after transfection/transduction, unless otherwise stated.

For Northern analysis, $1 \times 10^{5}$ Oct4-EGFP MEFs were transfected and harvested $5 \mathrm{~d}$ later. Total RNA was isolated by TRIZOL (Invitrogen) and $\sim 9 \mu \mathrm{g}$ of total RNA was resolved on a $14 \%$ denaturing polyacrylamide gel (National Diagnostics). RNAs were transferred onto Hybond-XL membranes (GE healthcare), and miRNAs were detected by isotopically labeled specific DNA probes. Signal intensity was visualized by phospho-imager and analyzed using Multi Gauge V3.0 (FUJIFILM). miRNA signal intensity was normalized to that of U6 snRNA. Experiments were performed in triplicate.

For Western analysis, $1 \times 10^{5}$ Oct4-EGFP MEFs were transfected and harvested $5 \mathrm{~d}$ later. Total proteins were prepared in M-PER buffer (Pierce), and equal amounts of total protein were separated on $10 \%$ SDS-PAGE gels. Proteins were transferred to PVDF membranes, and bands were detected using the following antibodies: GAPDH (Santa Cruz; catalog no. sc-20357), p53 (Santa Cruz; catalog no. sc-55476), PI3 kinase p85 (Cell Signaling; catalog no. 4257), Cdc42 (Santa Cruz; catalog no. sc-8401), p-ERK1/2 (Cell Signaling; catalog no. 9101), ERK1/2 (Cell Signaling; catalog no. 9102), p-GSK3 $\beta$ (Cell Signaling; catalog no. 9323), GSK3 $\beta$ (Cell Signaling; catalog no. 9315), and $\beta$-actin (Thermo Scientific; catalog no. MS-1295). Signal intensity was quantified by Multi Gauge V3.0 (FUJIFILM) and normalized to GAPDH or $\beta$-actin. Experiments were repeated three to five times.

\section{In vitro differentiation and teratoma formation assay}

For in vitro differentiation, iPSCs were dissociated by trypsin/ EDTA and resuspended in EB medium (DMEM with 15\% FBS, nonessential amino acid, L-glutamine) to a final concentration of $5 \times 10^{4}$ cells $/ \mathrm{mL}$. To induce EB formation, 1000 iPS cells in $20 \mu \mathrm{L}$ were cultured in hanging drops on inverted Petri dish lids. Three to $5 \mathrm{~d}$ later, EBs were collected and transferred onto $0.1 \%$ gelatincoated six-well plates at about 10 EBs per well. Two weeks after formation of EBs, beating cardiomyocytes (mesoderm) were identified by microscopy, and cells derived from endoderm and ectoderm were identified by $\alpha$-fetoprotein (R\&D; catalog no. MAB1368) and neuron-specific $\beta$ III-tubulin (abcam; catalog no. ab7751) antibodies, respectively. 
For teratoma assays, $1.5 \times 10^{6}$ iPSCs were trypsinized and resuspended in $150 \mu \mathrm{L}$ and then injected subcutaneously into the dorsal hind limbs of athymic nude mice anesthetized with avertin. Three weeks later, mice were killed to collect teratomas. Tumor masses were fixed, dissected, and analyzed in the Cell ImagingHistology core facility at the Sanford-Burnham Institute.

\section{Chimera analysis}

iPSC media was changed $2 \mathrm{hr}$ before harvest. Trypsinized iPSCs were cultured on $0.1 \%$ gelatin-coated plates for $30 \mathrm{~min}$ to remove feeder cells. iPSCs were injected into E3.5 C57BL/6-cBrd/cBrd blastocysts and then transferred into pseudopregnant recipient females. After birth, the contribution of iPSCs was evaluated by pup coat color: black is from iPSCs.

\section{Immunofluorescence and alkaline phosphatase staining}

iPSCs were seeded and cultured on $0.1 \%$ gelatin-coated six-well plates. Four days later, cells were fixed in $4 \%$ paraformaldehyde (Electron Microscopy Sciences; catalog no. 15710-S). For immunofluorescence staining, fixed cells were permeablized with $0.1 \%$ Trixton X-100 in PBS and blocked in 5\% BSA/PBS. Antibodies against SSEA-1 (R\&D; catalog no. MAB2155) and Nanog (R\&D; catalog no. AF2729) served as ES markers. Nuclei were visualized by Hoechst 33342 staining (Invitrogen). For alkaline phosphatase (AP) staining, fixed cells were treated with AP substrate following the manufacturer's instruction (Vector Laboratories; catalog no. SK-5100).

\section{SUPPLEMENTAL MATERIAL}

Supplemental material is available for this article.

\section{ACKNOWLEDGMENTS}

We thank the Rana laboratory members for helpful discussions and support. We thank the following shared resource facilities at the Sanford-Burnham Institute: genomics and informatics and data management core for miRNA and mRNA array experiments and data analysis, the flow cytometry facility, the animal facility for generation of teratomas and chimeric mice, and the histology and molecular pathology core for characterization of various tissues. This work was supported in part by NIH grants to T.M.R.

Received February 10, 2011; accepted May 5, 2011.

\section{REFERENCES}

Banito A, Rashid ST, Acosta JC, Li S, Pereira CF, Geti I, Pinho S, Silva JC, Azuara V, Walsh M, et al. 2009. Senescence impairs successful reprogramming to pluripotent stem cells. Genes Dev 23: 21342139.

Boyer LA, Lee TI, Cole MF, Johnstone SE, Levine SS, Zucker JP, Guenther MG, Kumar RM, Murray HL, Jenner RG, et al. 2005. Core transcriptional regulatory circuitry in human embryonic stem cells. Cell 122: 947-956.

Chang TC, Yu D, Lee YS, Wentzel EA, Arking DE, West KM, Dang CV, Thomas-Tikhonenko A, Mendell JT. 2008. Widespread
microRNA repression by Myc contributes to tumorigenesis. Nat Genet 40: 43-50.

Chang TC, Zeitels LR, Hwang HW, Chivukula RR, Wentzel EA, Dews M, Jung J, Gao P, Dang CV, Beer MA, et al. 2009. Lin-28B transactivation is necessary for Myc-mediated let-7 repression and proliferation. Proc Natl Acad Sci 106: 3384-3389.

Chen X, Xu H, Yuan P, Fang F, Huss M, Vega VB, Wong E, Orlov YL, Zhang W, Jiang J, et al. 2008. Integration of external signaling pathways with the core transcriptional network in embryonic stem cells. Cell 133: 1106-1117.

Chu CY, Rana TM. 2007. Small RNAs: regulators and guardians of the genome. J Cell Physiol 213: 412-419.

Dews M, Homayouni A, Yu D, Murphy D, Sevignani C, Wentzel E, Furth EE, Lee WM, Enders GH, Mendell JT, et al. 2006. Augmentation of tumor angiogenesis by a Myc-activated microRNA cluster. Nat Genet 38: 1060-1065.

Farh KK, Grimson A, Jan C, Lewis BP, Johnston WK, Lim LP, Burge CB, Bartel DP. 2005. The widespread impact of mammalian MicroRNAs on mRNA repression and evolution. Science 310: 1817-1821.

Geoghegan E, Byrnes L. 2008. Mouse induced pluripotent stem cells. Int J Dev Biol 52: 1015-1022.

Hong H, Takahashi K, Ichisaka T, Aoi T, Kanagawa O, Nakagawa M, Okita K, Yamanaka S. 2009. Suppression of induced pluripotent stem cell generation by the p53-p21 pathway. Nature 460: 11321135.

Ichida JK, Blanchard J, Lam K, Son EY, Chung JE, Egli D, Loh KM, Carter AC, Di Giorgio FP, Koszka K, et al. 2009. A small-molecule inhibitor of tgf- $\beta$ signaling replaces sox 2 in reprogramming by inducing nanog. Cell Stem Cell 5: 491-503.

Judson RL, Babiarz JE, Venere M, Blelloch R. 2009. Embryonic stem cell-specific microRNAs promote induced pluripotency. Nat Biotechnol 27: 459-461.

Kawamura T, Suzuki J, Wang YV, Menendez S, Morera LB, Raya A, Wahl GM, Belmonte JC. 2009. Linking the p53 tumour suppressor pathway to somatic cell reprogramming. Nature 460: 11401144.

Kim J, Chu J, Shen X, Wang J, Orkin SH. 2008. An extended transcriptional network for pluripotency of embryonic stem cells. Cell 132: 1049-1061.

Li H, Collado M, Villasante A, Strati K, Ortega S, Canamero M, Blasco MA, Serrano M. 2009. The Ink4/Arf locus is a barrier for iPS cell reprogramming. Nature 460: 1136-1139.

Li Z, Yang CS, Nakashima K, Rana TM. 2011. Small RNA-mediated regulation of iPS cell generation. EMBO J 30: 823-834.

Lin CH, Jackson AL, Guo J, Linsley PS, Eisenman RN. 2009. Mycregulated microRNAs attenuate embryonic stem cell differentiation. EMBO J 28: 3157-3170.

Liu G, Friggeri A, Yang Y, Milosevic J, Ding Q, Thannickal VJ, Kaminski N, Abraham E. 2010. miR-21 mediates fibrogenic activation of pulmonary fibroblasts and lung fibrosis. J Exp Med 207: 1589-1597.

Marion RM, Strati K, Li H, Murga M, Blanco R, Ortega S, FernandezCapetillo O, Serrano M, Blasco MA. 2009. A p53-mediated DNA damage response limits reprogramming to ensure iPS cell genomic integrity. Nature 460: 1149-1153.

Mayr C, Bartel DP. 2009. Widespread shortening of 3'UTRs by alternative cleavage and polyadenylation activates oncogenes in cancer cells. Cell 138: 673-684.

Melton C, Judson RL, Blelloch R. 2010. Opposing microRNA families regulate self-renewal in mouse embryonic stem cells. Nature 463: 621-626.

Nakagawa M, Koyanagi M, Tanabe K, Takahashi K, Ichisaka T, Aoi T, Okita K, Mochiduki Y, Takizawa N, Yamanaka S. 2008. Generation of induced pluripotent stem cells without Myc from mouse and human fibroblasts. Nat Biotechnol 26: 101-106.

Nichols J, Silva J, Roode M, Smith A. 2009. Suppression of Erk signalling promotes ground state pluripotency in the mouse embryo. Development 136: 3215-3222. 
Okita K, Ichisaka T, Yamanaka S. 2007. Generation of germlinecompetent induced pluripotent stem cells. Nature 448: 313-317.

Park SY, Lee JH, Ha M, Nam JW, Kim VN. 2009. miR-29 miRNAs activate p53 by targeting p85 alpha and CDC42. Nat Struct Mol Biol 16: 23-29.

Pelengaris S, Khan M, Evan G. 2002. c-MYC: more than just a matter of life and death. Nat Rev Cancer 2: 764-776.

Rana TM. 2007. Illuminating the silence: understanding the structure and function of small RNAs. Nat Rev Mol Cell Biol 8: 23-36.

Seifinejad A, Tabebordbar M, Baharvand H, Boyer LA, Salekdeh GH. 2010. Progress and promise towards safe induced pluripotent stem cells for therapy. Stem Cell Rev 6: 297-306.

Seoane J, Pouponnot C, Staller P, Schader M, Eilers M, Massague J. 2001. TGF $\beta$ influences Myc, Miz-1 and Smad to control the CDK inhibitor p15INK4b. Nat Cell Biol 3: 400-408.

Seoane J, Le HV, Massague J. 2002. Myc suppression of the p21(Cip1) Cdk inhibitor influences the outcome of the p53 response to DNA damage. Nature 419: 729-734.

Silva J, Barrandon O, Nichols J, Kawaguchi J, Theunissen TW, Smith A. 2008. Promotion of reprogramming to ground state pluripotency by signal inhibition. PLoS Biol 6: e253. doi: 10.1371/journal.pbio.0060253.

Smith KN, Singh AM, Dalton S. 2010. Myc represses primitive endoderm differentiation in pluripotent stem cells. Cell Stem Cell 7: 343-354.

Sridharan R, Tchieu J, Mason MJ, Yachechko R, Kuoy E, Horvath S, Zhou Q, Plath K. 2009. Role of the murine reprogramming factors in the induction of pluripotency. Cell 136: 364-377.
Takahashi K, Yamanaka S. 2006. Induction of pluripotent stem cells from mouse embryonic and adult fibroblast cultures by defined factors. Cell 126: 663-676.

Takahashi K, Tanabe K, Ohnuki M, Narita M, Ichisaka T, Tomoda K, Yamanaka S. 2007. Induction of pluripotent stem cells from adult human fibroblasts by defined factors. Cell 131: 861-872.

Thum T, Gross C, Fiedler J, Fischer T, Kissler S, Bussen M, Galuppo P, Just S, Rottbauer W, Frantz S, et al. 2008. MicroRNA-21 contributes to myocardial disease by stimulating MAP kinase signalling in fibroblasts. Nature 456: 980-984.

Utikal J, Polo JM, Stadtfeld M, Maherali N, Kulalert W, Walsh RM, Khalil A, Rheinwald JG, Hochedlinger K. 2009. Immortalization eliminates a roadblock during cellular reprogramming into iPS cells. Nature 460: 1145-1148.

Wanzel M, Herold S, Eilers M. 2003. Transcriptional repression by Myc. Trends Cell Biol 13: 146-150.

Ying QL, Wray J, Nichols J, Batlle-Morera L, Doble B, Woodgett J, Cohen P, Smith A. 2008. The ground state of embryonic stem cell self-renewal. Nature 453: 519-523.

Yoshida Y, Yamanaka S. 2010. Recent stem cell advances: induced pluripotent stem cells for disease modeling and stem cell-based regeneration. Circulation 122: 80-87.

Yu J, Vodyanik MA, Smuga-Otto K, Antosiewicz-Bourget J, Frane JL, Tian S, Nie J, Jonsdottir GA, Ruotti V, Stewart R, et al. 2007. Induced pluripotent stem cell lines derived from human somatic cells. Science 318: 1917-1920. 

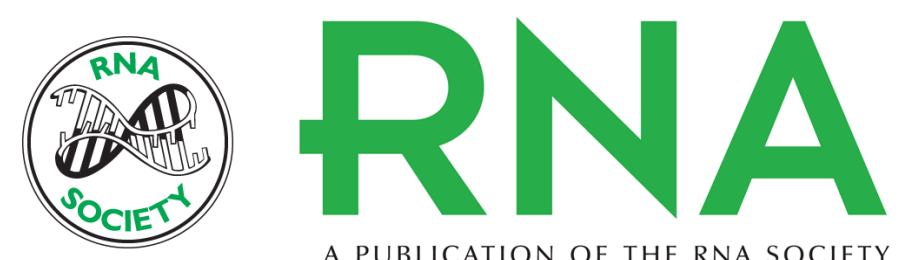

A PUBLICATION OF THE RNA SOCIETY

\section{microRNAs modulate iPS cell generation}

Chao-Shun Yang, Zhonghan Li and Tariq M. Rana

RNA 2011 17: 1451-1460 originally published online June 21, 2011

Access the most recent version at doi:10.1261/rna.2664111

Supplemental http://rnajournal.cshlp.org/content/suppl/2011/06/01/rna.2664111.DC1

References This article cites 41 articles, 7 of which can be accessed free at: http://rnajournal.cshlp.org/content/17/8/1451.full.html\#ref-list-1

Open Access Freely available online through the RNA Open Access option.

License Freely available online through the RNA Open Access option.

Email Alerting Receive free email alerts when new articles cite this article - sign up in the box at the Service top right corner of the article or click here.

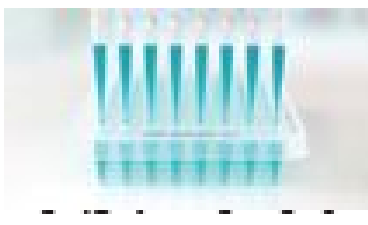

Providing Precise Solutions for your research.

To subscribe to RNA go to:

http://rnajournal.cshlp.org/subscriptions 